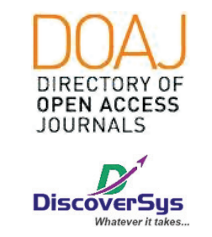

Published by DiscoverSys

\title{
The characteristic of tinea capitis in children among dermato-venereology outpatients' polyclinic at Sanglah General Hospital, Denpasar from 2014- 2017: A preliminary study
}

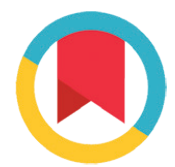

CrossMark

\author{
Velava Kumar Gopal, ${ }^{1 *}$ Made Swastika Adiguna, ${ }^{2}$ \\ Prima Sanjiwani Saraswati Sudarsa
}

\section{ABSTRACT}

Background: Tinea capitis is a type of dermatophytosis (skin disease) caused by the infection of dermatophytes. It is generally caused by the dermatophyte species of Microsporum and Trichophyton. It has been known to be able to spread human to human and animal to human. It is also almost commonly found in the prepubertal age and very rare among the adults of either gender. This study aims to determine the prevalence of Tinea capitis in children by age, sex, clinical manifestation, laboratory tests, and treatments.

Methods: A cross-sectional observational study from the medical records of patients with Tinea capitis was conducted at DermatoVenereology Outpatient Clinic RSUP Sanglah from 2014 to 2017. A total of 5 research variables were identified for this study: age, sex, clinical manifestation, laboratory tests, and treatment. The data was then analyzed and interpreted and further presented in forms of description, tables and graphs in this study by using SPSS version 17 for Windows

Results: A total of 32 patients were identified as having Tinea capitis from the period of 2014-2017. There was a slightly higher prevalence in the male gender (56.25\%) compared with female gender (43.75\%). The highest prevalence of the disease was found in the age groups of $1-5$ and $6-10$ years old with $43.75 \%$ respectively. Majority of the patients showed the presence of grey patch on their scalp on examination (53.13\%). The dermatophyte Trichophyton rubrum had the highest isolation in comparison with other dermatophytes at $25.00 \%$. The patients were almost all treated with oral Griseofulvin. Conclusion: The majority diagnosis of Tinea capitis was found in group 1-5 and 6-10 years old and had a grey patch appearance, whereas Trichophyton rubrum as the highest isolation in comparison with other dermatophytes

Keywords: Tinea capitis, characteristics, clinical manifestation, outpatients.

Cite This Article: Gopal, V.K., Adiguna, M.S., Sudarsa, P.S.S. 2019. The characteristic of tinea capitis in children among dermato-venereology outpatients' polyclinic at Sanglah General Hospital, Denpasar from 2014-2017: A preliminary study. Intisari Sains Medis 10(3): 563-567. D0I: 10.15562/ism.v10i3.461

\section{INTRODUCTION}

Dermatophytosis is the infection of the skin (tinea pedis), hair (tinea capitis), or nail (onychomycosis) caused specifically by dermatophytes and can be distinguished by skin turning reddish, and appearance of small papular vesicles, fissures and scaling. ${ }^{1}$ Dermatophytosis is caused by the fungi under the genera of Microsporum, Trichophyton, and Epidermophyton. ${ }^{2}$ They are generally called as dermatophytes or in certain publishing as dermatophytosis if they are known to be soil-borne. These dermatophytes are characterized by their ability to be able to digest the keratin found in tissues like the epidermis, hair, nails, feathers, horns, and hooves. The infection of the scalp is specifically known as Tinea capitis. ${ }^{2}$

Tinea capitis remains a major public health issue, particularly in a developing country which is generally characterized by a high population but unable to meet all the basic needs for a good quality of life. ${ }^{3}$ Studies have found that of all the dermatological cases studied; there is a $20 \%$ occurrence of dermatomycosis with conditions due to dermatophyte infections as the most seen at $11.4 \% .^{4} \mathrm{~A}$ further statistical breakdown provided by studies that have been published shows that tinea capitis itself is highly common to those of 6-10 years of age, school-attending and prepubertal.

Tinea capitis, which is more commonly found in children of the school-going age and prepubertal, is infectious. It is caused by the Microsporum sp. and Trichophyton sp. It is known to project both anthropophilic infections (child to child) and zoophilic infection (animals to children). ${ }^{5}$ Due to these, it is highly infectious particularly to children when they are carrying out their daily activities like attending school and playing with common pets such as 
dog and cats. ${ }^{6}$ Tinea capitis has multiple treatment options that are proven to be effective though it is known to take up to 1 month for the results to be seen. However, the lack of assimilation, together with a systematic approach to control through case ascertainment and therapy has allowed the disease to continue to be a public healthcare burden. ${ }^{2}$

Based on the aforementioned, this study aims to determine the characteristics of tinea capitis on several aspects such as age, sex, clinical manifestations, laboratory tests: $\mathrm{KOH}$, Wood lamp and Culture and the given course of treatment.

\section{METHODS}

A retrospective cross-sectional observational study was conducted among 32 patients using secondary data at Dermato-Venereology Outpatient Clinic RSUP Sanglah in Denpasar from the period January 2014 until December 2017. The inclusion criteria were: 1 ) The target population of the study are patients with Tinea capitis, and 2) The sampled population of the study are those patients below the age of 21 years until December 2017. The sampling technique used in this study was non-probability purposive sampling. Several parameters were assessed in this study such as age, gender, clinical manifestation, and laboratory test using $\mathrm{KOH}$, Woods lamp, and culture examination.

Data for this study will be collected as part of a descriptive study using data that has already been provided and recorded. The data were collected from Patient Registration Logbook of all patients with tinea capitis, aged below 21 years old and will be analyzed using SPSS version 17 for Windows.

\section{RESULTS}

A total number of 9 cases of Tinea capitis were recorded representing $28.13 \%$ in percentage terms of the total cases in 4 consecutive years. In 2015, 10 cases $(31.25 \%)$ were recorded, showing an increment compared to the year before. For the year 2016, a total of 9 cases were recorded showing a decrease in Tinea capitis case from the years before and representing $28.13 \%$ of total cases in the 4 years being studied in this study. For the 12 months of 2017 , only 4 cases (12.49\%) were recorded beings the least number of cases being reported in the 4 consecutive years of $2014-2017$. The following graph shows the distribution of prevalence of Tinea capitis in children in RSUP Sanglah 2014 - 2017 (Figure 1).

In this study, it only involves cases of Tinea capitis in children ranging from the age of 1-year-old to 21 years old. From the 32 recorded patients in RSUP Sanglah from 2014 - 2017, there were 14 cases
Table 1 The prevalence of Tinea capitis based on age, sex, clinical manifestation, $\mathrm{KOH}$ examination, and wood lamp examination in children at RSUP Sanglah

Parameter Amount Percentage (\%)

Age

$$
\begin{aligned}
& 1-5 \text { years } \\
& 6-10 \text { years } \\
& 11-15 \text { years } \\
& 16-21 \text { years }
\end{aligned}
$$

Sex

Male

Female

Clinical

Manifestation

Grey patch

Kerion

Black dot

Favus

Alopecia areata

Grey patch/black

dot

Kerion/favus

43.75

43.75

6.25

6.25

KOH examination

Ectothrix spores

Endothrix spores

Negative

Ectothrix/

Endothrix

Pseudohyphae

Not tested

Wood lamp

examination

Greenish-yellow
Green

40.63

25.00

Yellowish

Bluish-green

3.12

6.25

Negative

25.00

Culture examination

$\begin{array}{llc}\text { T. rubrum } & 8 & 25.00 \\ \text { T. } \text { tonsurans } & 5 & 15.63 \\ \text { T. mentagrofit } & 5 & 15.63 \\ \text { T. violaceum } & 1 & 3.12 \\ \text { M. canis } & 3 & 9.38 \\ \text { M. andouinii } & 3 & 9.38 \\ \text { Negative } & 3 & 9.38 \\ \text { Not tested } & 4 & 12.50\end{array}$




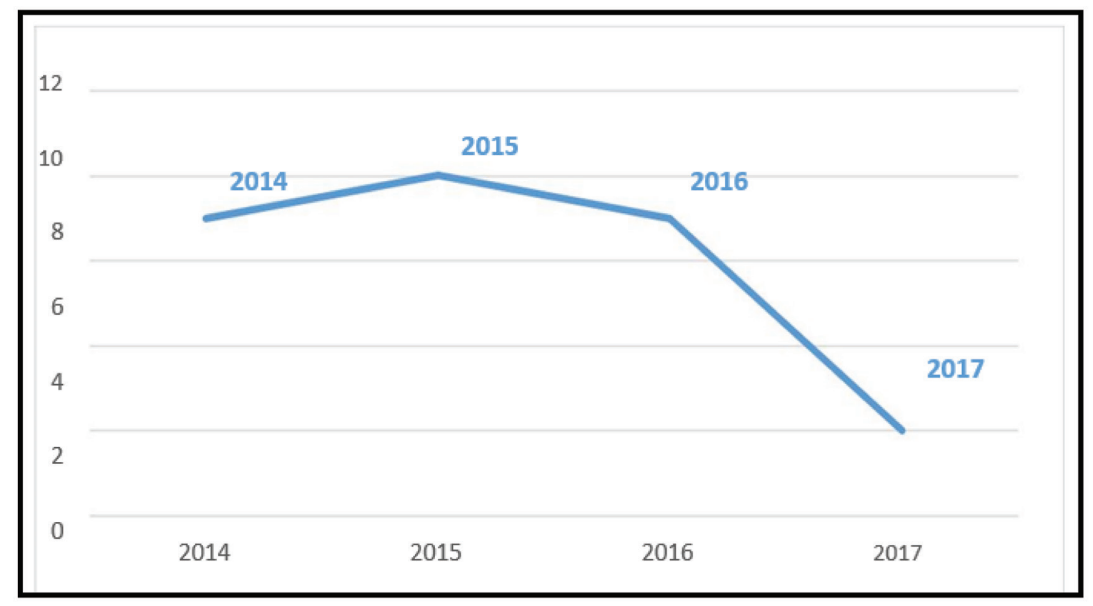

Figure 1 The prevalence of Tinea capitis in children during 2014-2017

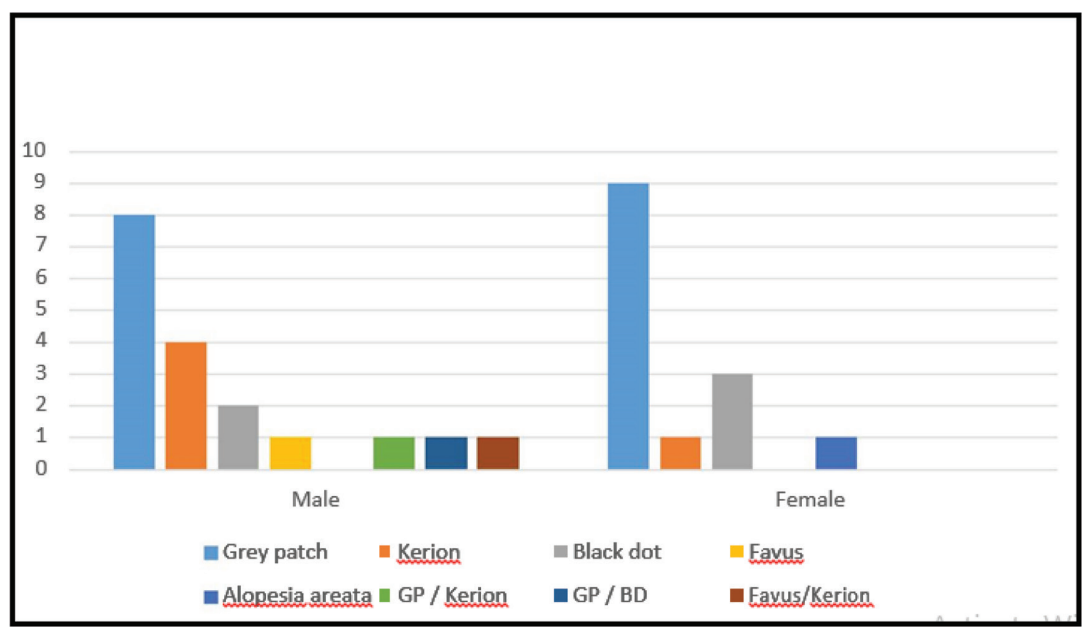

Figure 2 The prevalence of Tinea Capitis based on Clinical Manifestation and Gender

recorded with the age range of 1 year to 5 years old representing $43.75 \%$. Of these 14 months old: 1 case, 2 years old: 1 case, 3 years old: 4 cases, 4 years old: 4 cases, and 5 years old: 4 cases. Total of 14 cases was recorded in $6-10$ years old range representing $43.75 \%$. For the age ranges of $11-15$ years old and 16 - 21 years old, there were 2 cases for each equating to $6.25 \%$, respectively (Table 1 ).

Of the 32 recorded patients with Tinea capitis from 2014 - 2017 in RSUP Sanglah, there were more male cases recorded compared with female cases. A total of 18 cases recorded were of male dominance, while only 14 were of females. These corresponded to $56.25 \%$ male cases and $43.75 \%$ female cases (Table 1). Of the 32 recorded patients with Tiena is from 2014 - 2017 in RSUP Sanglah, the majority of the patients showed the presence or manifestation of tinea capitis with a grey patch. A total of 18 patients presented with this manifestation equaling to $56.25 \%$ of the total patients. A total of 5 patients presented with tinea capitis with kerion and tinea capitis with black dot respectively. These represented $15.63 \%$ of the total for the respective clinical manifestation. For the following clinical manifestation there was single patient for each: tinea capitis with favus 1 patient, tinea capitis with grey patch and black dot 1 patient, Tinea capitis with kerion and favus 1 patient representing 3.122\% respectively of the total. A further single patient had tinea capitis with alopecia areata. A single patient also represented with secondary infection kerion while the primary infection showed a grey patch.

From the 32 recorded patients with Tinea capitis from 2014 - 2017 in RSUP Sanglah 18 were male patients and 14 were female patients. To compare with the clinical manifestation: the majority of the patients had Tinea capitis with a grey patch at 17 patients. Of these, 8 patients were male, while 9 were female patients presenting a gray patch. Second, 5 overall pateints were showing the characteristic of Tinea capitis with kerion. Of these, 4 were male patients, and 1 was a female patient. For Tinea capitis with a black dot, 3 patients were female while 2 were male patients. There was a single male patient with favus. There was also a single female patient with alopecia areata. There were also single male patients for tinea capitis with grey patch and kerion; grey patch and black dot; favus and kerion, respectively (Figure 2).

Of the 32 recorded patients with Tinea capitis from 2014 - 2017 in RSUP Sanglah, the majority of the patients had laboratory test: $\mathrm{KOH}$ examination done. Twenty-eight patients of the total had this examination while the remaining 4 patients did not. The outcome of the test is classified as follows: the presence of spores either ectothrix, endothrix or mixed, presence of pseudohyphae and negative outcome or lack of spore presentation. The majority of the patients tested positive for the presence of spores in the ectothrix pattern: 15 patients representing 46.88\%. A further 7 patients $(21.88 \%)$ had a negative outcome for this test. A total of 4 patients presented with endothrix pattern equating with $12.50 \%$. A single patient presented with both ectothrix and endothrix spores pattern. There was also 1 patient $(3.12 \%)$ with pseudo hyphae (Table 1 ).

Of the 32 recorded patients with Tinea capitis from 2014 - 2017 in RSUP Sanglah, all the patients had laboratory test: Wood lamp examination is done. The presence of spores will cause the hair to fluoresce under this UV light if its ectothrix infection. Of the 32 patients tested a total of 8 patients tested negative, or the hair did not show any fluoresce. This represented $25.0 \%$ of the total. A further 13 patients fluoresced in this test with a greenish-yellow color. This represented $40.63 \%$ of the total. Another eight patients (25.0\%) showed positive for this test with the hair fluorescing green 
color. Two patients showed bluish-green color equating $6.25 \%$ of the total. A single patient fluoresced a yellowish color in the test representing $3.12 \%$ (Table 1).

Of the 32 recorded patients with Tinea capitis from $2014-2017$ in RSUP Sanglah majority of the patients had laboratory test: culture examination is done to identify the dermatophyte causing the disease. Of the 32 patients, only 4 patients were not tested with culture examination. A total of 8 patients (25.0\%) tested positive for the culture test showing the presence of Tr. rubrum as the causing dermatophyte. About 5 patients were showing the presence of Tr. tonsurans are representing 15.63\% of the total. A further 5 patients (15.63\%) tested positive for Tr. mentagrofit as the causative dermatophyte. Of the total patients, 3 patients showed the presence of $M$. canis as the causative agent equating for $9.38 \%$ of the total. A further 3 patients tested positive for $M$. andouinii, also representing $9.38 \%$ of the total. A single patient tested positive for Tr. violaceum as the causing dermatophyte. A total of 3 patients were tested for this examination but found to be of negative result meaning there was no presence of dermatophytes representing 9.38\% (Table 1).

Of the 32 recorded patients with Tiena capitis from 2014 - 2017 in RSUP Sanglah all the patients were treated according to the treatment module of RSUP Sanglah Dermato-Venereology Outpatient Clinic, a total of 29 patients were given Griseofulvin per oral and a single patient received Amoxicillin per oral. All 32 patients were also treated with topical medications such as Ketoconazole 2\% shampoo, and some were given $\mathrm{NaCl} 0.9 \%$ compress as a topical treatment.

\section{DISCUSSION}

Tinea capitis is a fungal infection of the skin that affects the scalp, eyebrow, and eyelash. It is a common infectious skin disease of children. It is caused by dermatophytes particularly those of the Microsporum sp. and Trichophyton sp. Individuals with Tinea capitis often show the characterization of spreading, scaly, irregular or well-demarcated areas of erythema and alopecia. Goldsmith et al. said Tinea capitis is most commonly observed in the age range of 3 years to 10 years old with a higher number of occurrences in the male gender. ${ }^{7}$

Among the children in Dermato-Venereology Outpatient Clinic RSUP Sanglah from 2014-2017, a pattern for age is observable. There were 32 cases recorded for this period for children having Tinea capitis. There were 14 patients (43.75\%) in the age range of 0-5 years old. Another 14 patients were from the age range of 6-10 years old. There were 2 patients (6.25\%) each from the age range of 11-15 years old and 16-21 years old. Most of the patients with Tinea capitis prevailed from those of under 10 years old. Based on the study by Moto et al. found that out of 150 children examined a total of 122 had symptoms of dermatophyte infection. ${ }^{8}$ This equates to a prevalence rate of $81.3 \%$ among children. To compare with other published studies, there were a total of 26 patients with Tinea capitis that were within the age range of 3-10 years old, representing $81.25 \%$ of the total patients recorded.

Prevalence of Tinea capitis in DermatoVenereology Outpatient Clinic in RSUP Sanglah from the year 2014 - 2017 showed a record of 32 patients. When broken down by gender or sex, there were 18 male patients and 14 female patients. This represents a $58.62 \%$ to $41.38 \%$ male to female prevalence. This corresponds with studies such as Moto et al., which states that Tinea capitis prevalence is higher in male than female. ${ }^{8}$ This could be since hormones such as progesterone may play a part as inhibitory on dermatophyte growth for the female gender. It could also be due to the more active daily activities of the male gender, such as physical playing with friends allowing for more human-human infections to take place.

Among the recorded patients, the most common identified dermatophyte was the anthropophilic Trichophyton rubrum corresponding similarly with a study from Nigerian school children by Dogo et al. ${ }^{9}$ About 8 patients tested positive for the presence of this organism as the causative organism. Both Trichophyton tonsurans and Trichophyton mentagrofit were isolated and identified from 5 patients each. Other dermatophytes that were identified were Trichophyton violaceum, Microsporum canis, and Microsporum andouinii. Tr. violaceum was prevalent at $3.12 \%$ or a single patient, $M$. canis had 3 patients at $9.38 \%$ and 3 patients with M. andouinii. These could have been contributed by lack of hygiene practices in activities of daily living. For example, the sharing of combs, towels, and helmets as well as not washing the hair often enough to keep it clean. This could be due to factors such as inappropriate sampling, contamination of the culture, and other medium factors.

In this study, it was observed that the patients were almost universally treated with oral Griseofulvin and topical Ketoconazole 2\% shampoo. Amoxycillin per oral was given to one patient after being identified as having a secondary infection due to Tinea capitis. The British Association of Dermatologists produced a leaflet on Tinea capitis 
in August 2014 and further updated it in November 2017 states that Tinea capitis is a curable disease using oral antifungal medication and antifungal shampoo. They further operationalize the usage of two types of antifungal drugs Griseofulvin species and Terbinafine per oral. However, only the usage of Griseofulvin is licensed while the usage of Terbinafine is not licensed in children. However, in terms of effectiveness, it is known that Terbinafine is the choice of medicine. The leaflet further gives the option of itraconazole and fluconazole. A previous study also stated that based on 3 case studies using oral Griseofulvin and topical Ketoconazole $2 \%$ shampoo showed improvement for patients without side effects being observed. ${ }^{10}$

\section{CONCLUSION}

This study found that the greatest number of patients with Tinea capitis in the Dermato-Venereology Outpatient Clinic RSUP Sanglah hailed from the age groups of $0-5$ years old and $6-10$ years old with 14 patients respectively. In addition, it was also found that males were dominant than the females from January 2014 to December 2017. Majority of the patients in the Dermato-Venereology Outpatient Clinic RSUP Sanglah during the study period were found to have a grey patch on the scalp. The recent study also found that the most commonly isolated and identified organism was Trichophyton rubrum. In general, the treatment for these patients in the Dermato-Venereology Outpatient Clinic RSUP Sanglah was given oral Griseofulvin.

\section{CONFLICT OF INTEREST}

The authors declare that there is no competing interest regarding the manuscript.

\section{ETHICS CONSIDERATION}

Ethics approval has been obtained from the Ethics Committee of Faculty of Medicine, Universitas Udayana prior to the study being conducted.

\section{FUNDING}

The authors are responsible for the funding of study without the involvement of grant, scholarship, or sponsorship.

\section{AUTHOR CONTRIBUTIONS}

All of authors are equally contribute to the study from a conceptual framework, data gathering, data analysis, until reporting the results of study.

\section{REFERENCE}

1. Gupta AK, Ryder JE, Chow M, Cooper EA. Dermatophytosis: the management of fungal infections. Skinmed. 2005;4(5):305-10.

2. Hay RJ. Tinea Capitis: Current Status. Mycopathologia. 2017;182(1-2):87-93

3. Tan CW, Foong AY, Liew HM, Koh MJ. A Review of Tinea Capitis in a Cohort of Asian Children. Ann Acad Med Singapore. 2018 Apr;47(4):156-158.

4. Sukmara IM, Miliawati R, Sukandar H. Dermatomycosis among Elementary School Children in Jatinangor West Java. Althea Medical Journal. 2015;2(1):268-75

5. Jamison DT, Breman JG, Measham AR, et al., editors. Disease Control Priorities in Developing Countries. 2nd edition. Washington (DC): The International Bank for Reconstruction and Development / The World Bank; 2006. Available from: https://www.ncbi.nlm.nih.gov/books/ NBK11728/ Co-published by Oxford University Press, New York.

6. Gupta S, Purva A, Rahul R, Saksham G. Prevalence of Dermatophytic Infection and Determining Sensitivity of Diagnostic Procedures. International Journal of Pharmacy and Pharmaceutical Sciences. 2014;6(3):35-38

7. Goldsmith LA, Katz SI, Gilchrest BA. Paller AS, Leffell DJ, Wolff $\mathrm{K}$, et al. Fitzpatrick's Dermatology in General Medicine. $8^{\text {th }}$ Edition. New York: The McGraw-Hill Companies, Inc. 2012:2277-2334

8. Moto JN, Maingi JM, Nyamache AK. Prevalence of Tinea capitis in school going children from Mathare, informal settlement in Nairobi, Kenya. BMC Res Notes. 2015;8:274.

9. Dogo J, Afegbua SL, Dung EC. Prevalence of Tinea Capitis among School Children in Nok Community of Kaduna State, Nigeria. J Pathog. 2016; 2016: 9601717.

10. Greer DL. Successful treatment of tinea capitis with $2 \%$ ketoconazole shampoo. Int J Dermatol. 2000;39(4):302-4.

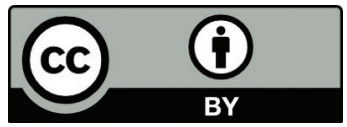

This work is licensed under a Creative Commons Attribution 